\title{
The Fault Diagnosis of Rolling Bearing based on MED and HHT
}

\author{
Zhidong Wang \\ School of Mechanical Engineering, Shandong \\ University, \\ Key Laboratory of High Efficiency and Clean \\ Mechanical Manufacture, Shandong University, \\ Jinan, China \\ e-mail:wzdhbdjk@163.com
}

\author{
Rui Huo \\ School of Mechanical Engineering, Shandong \\ University, \\ Key Laboratory of High Efficiency and Clean \\ Mechanical Manufacture, Shandong University, \\ Jinan, China \\ e-mail:huorui@sdu.edu.cn
}

\author{
Daokun Zhang \\ School of Mechanical Engineering, Shandong \\ University, \\ Key Laboratory of High Efficiency and Clean \\ Mechanical Manufacture, Shandong University, \\ Jinan , China \\ e-mail: zhangdaokun1030@136.com
}

\begin{abstract}
As an essential part of rotating machinery, the early fault diagnosis of rolling bearing can improve the safety of mechanical operation. What's more, the machining accuracy can be effectively ensured. Unfortunately, the early fault signal of rolling bearing is extremely weak, which can be easily covered by others. And the endpoint effect will appear in several Intrinsic Mode functions (IMFs) when it is decomposed by Hilbert-Huang transform (HHT), which make it difficult to find the fault position accurately. To get the real components, the minimum Entropy Deconvolution (MED) method is proposed here to obtain the effective impact components with an inverse filter, which can improve the signal-to-noise ratio. The MED method which can effectively extract the useful information of the rolling bearing's fault signal, and fully suppress the endpoint effect of Empirical Mode decomposition (EMD), as well as greatly improve the ability to precisely find the fault position, which has been confirmed by a lot of experiments, which has a great practical value in the practical production.
\end{abstract}

Keywords- Rolling bearing; optimal filter; Minimum entropy deconvolution; HHT; Fault Diagnosis;

\section{INTRODUCTION}

According to a recent survey, it show that almost $30 \%$ [1] of mechanical failure during the operation process of rotating machinery caused by rolling bearing's fault. So it is of great significance to conduct the early fault diagnosis, through which safe operation and precision can be effectively guaranteed. Fault diagnosis contains two parts, one is feature components extraction and the other is fault identification. As all known, some useful methods such as fast spectral kurtosis [2-3], wavelet transform [4-5] have been widely used in feature extraction. But they can only effectively extract the fault signal of rolling bearing components on a certain degree. When it comes to strong noise background signals, they have a poor performance on fault component extraction. So the subsequent fault can't be accurately identified.

HHT [6-8] is an adaptive signal processing method based on local signal characteristics, consisted of two parts: Firstly, preprocessed signal decomposed by empirical mode decomposition (EMD) method into intrinsic mode function (IMF) series. Secondly, fault position identified according to its Hilbert spectrum. What troubles us most is that endpoint effect appears in the first and second IMFs decomposed by EMD, from which the fault position can't be found from their Hilbert spectrum. In order to solve the problem, minimum entropy deconvolution (MED) [10-11] which can effectively extract the spike pulse of weak signal proposed here to preprocess the early fault signal of rolling bearing collected, then real signal obtained. Combined with HHT method, the fault position can be exactly found.

\section{BRIEF INTRODUCTION OF MINIMUM ENTROPY DECONVOLUTION}

Minimum entropy deconvolution firstly proposed by Wiggins in 1978[12] to deal with the seismic reflection data, then applied in gear fault diagnosis by Endo. MED is an effect way to get the real signal from the signal acquired by looking for the right inverse filter.

Assuming that the system input $\mathrm{x}(\mathrm{k})$ is the sparse spike pulse sequence, which has simple characteristics. When it comes to a certain deterministic system, the entropy is smaller. Then output obtained:

$\mathrm{z}(\mathrm{k})=\mathrm{h}(\mathrm{k}) * \mathrm{x}(\mathrm{k})$

The signal will lose the characteristics of simple and certainty when it goes through the system, owing to the entropy increases clandestinely. The fundamental of MED 
is to look for a inverse filter $\mathrm{g}(\mathrm{l})$ on $\mathrm{L}$ order, through which the output $y(k)$ could be restore the system's input $x(k)$, that's is:

$$
y(\mathrm{k})=\sum_{k=1}^{L} g(\mathrm{l}) \mathrm{z}(\mathrm{k}-\mathrm{l}) \approx \beta x(\mathrm{k}-\tau)
$$

In order to get the characteristics of simple and certainty again, time delay and amplitude are allowed when get the real $\mathrm{x}(\mathrm{k})$ based on $\mathrm{y}(\mathrm{k})$, and the entropy decreases.

With the purpose of designing a optimal filter $\mathrm{g}(\mathrm{l})$ to make the entropy smaller, the objective function(equation(3)) equals zero(equation(4))

$$
\mathrm{O}_{4}(\mathrm{~g}(\mathrm{l}))=\sum_{i=1}^{N} y^{4}(\mathrm{i}) /\left[\sum_{i=1}^{N} y^{2}(\mathrm{i})\right]^{2}
$$$$
\partial\left(\mathrm{O}_{4}(\mathrm{~g}(\mathrm{l}))\right) / \partial(\mathrm{g}(\mathrm{l}))=0
$$

Then we obtain:

$$
\left[\sum_{k=1}^{N} y^{2}(\mathrm{k}) / \sum_{k=1}^{N} y^{4}(\mathrm{k})\right] \sum_{k=1}^{N} y^{3}(\mathrm{k}) z(\mathrm{k}-\mathrm{l})=\sum_{p=1}^{L} g(\mathrm{p}) \sum_{k=1}^{N} z(\mathrm{k}-\mathrm{i}) \mathrm{z}(\mathrm{k}-\mathrm{p})
$$

When it meets equation(5), MED method can effectively capture the spike pulse components in the early and weak fault signal of rolling bearing. What excites us most is that shock signal can be restored perfectly.

\section{HILBERT-HUANG TRANSFORM}

Hilbert-Huang Transform mainly divided into two steps: signal decomposition and Hilbert spectrum obtention. Decompose signal to get IMFs is an important part of the HHT, and each IMF meets two requirements: (1) the number of poles and zeros' difference should be equals zero or one at most in the whole data. (2)The average value of envelope gained from the absolute maximum of upper envelope and lower envelope must be zero at any point, that's the upper envelope and lower envelope must be axisymmetric.

After first step, the relationship between signal components obtained:

$$
x(\mathrm{t})=\sum_{j=1}^{n} c_{j}+r_{n}
$$

Where $x(t)$ is the signal processed by EMD, $c_{j}$ means IMF components, $r_{n}$ means residual component after decomposition. And limited standard deviation (SD) chosen here to judge the whether the IMF obtention should come to an end or not. In this paper, the SD value ranges from 0.2 to 0.3 .

The SD calculated from two consecutive IMFs chosen, just like equation (7).

$$
S D=\sum_{t=0}^{T} \frac{\left[h_{k-1}(\mathrm{t})-\mathrm{h}_{k}(\mathrm{t})\right]^{2}}{h_{k-1}^{2}(\mathrm{t})}
$$

\section{ANALYSIS OF FAULT DATA COLLECTED}

In this section, the methods mentioned above have been applied to deal with the fault data collected to find the accurate fault position of rolling bearing. First of all, the MED used to get the real shock signal, then EMD to get the IMF series. Correlation coefficient applied to select some appropriate IMFs, and their real failure frequency will be obtained by Hilbert spectrum. Compared with their theoretical failure frequency, the fault position will be identified easily (Figure 1.).

The geometric parameters of fault rolling bearing tested listed in Tab.1, and the theoretical failure frequency calculation formulas as follow(equation(8) and (9)).

TABLE I. GEOMETRIC PARAMETERS OF FAULT ROLLING BEARING TESTED

\begin{tabular}{cccc}
\hline $\begin{array}{c}\text { Pitch diameter } \\
(\mathbf{m m})\end{array}$ & $\begin{array}{c}\text { diameter } \\
(\mathbf{m m})\end{array}$ & $\begin{array}{c}\text { elements } \\
\text { number }\end{array}$ & $\begin{array}{c}\text { contact } \\
\text { angle }(\mathbf{o})\end{array}$ \\
\hline 38.5 & 7.985 & 7.985 & 0 \\
\hline
\end{tabular}

$f_{\text {outer }}=\frac{z}{2}\left(1-\frac{d}{D} \cos \alpha\right) f_{r}$

$f_{\text {inner }}=\frac{z}{2}\left(1+\frac{d}{D} \cos \alpha\right) f_{r}$

Where the $\mathrm{D}$ means pitch diameter, $\mathrm{d}$ means diameter, n means elements number, $\alpha$ means contact angle. $\mathrm{fr}=\mathrm{N} / 60$, the $\mathrm{N}$ refers the input shaft's rotation.

When the speed $\mathrm{N}$ equals $800 \mathrm{r} / \mathrm{min}$, the inner and outer theoretical failure frequency obtained by equation (8) and (9), finner $=72.4442 \mathrm{~Hz}$, fouter $=47.5558 \mathrm{~Hz}$.

\section{A. $\quad$ Outer ring fault diagnosis}

The fault signal collected (Figure 2.) of outer ring, which has been preprocessed by MED mentioned above shown in Figure 2. Comparing with Figure 2, the obvious shock components can be found easily, which means the MED can effectively restore te weak signal.

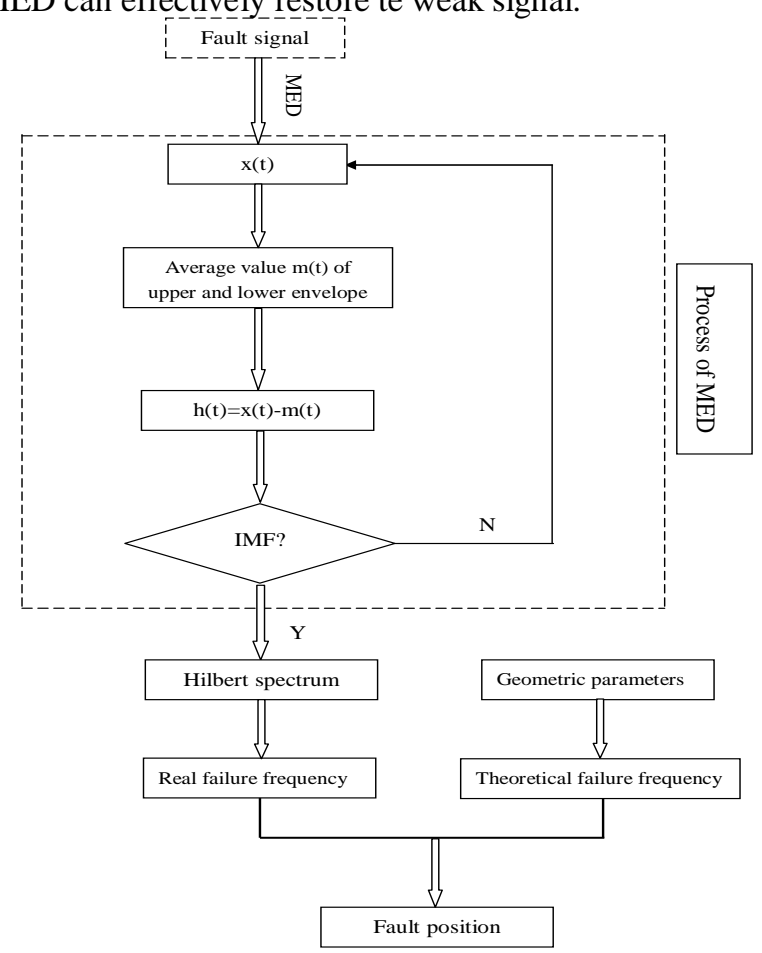

Figure 1. Specific flow chart of fault diagnosis 


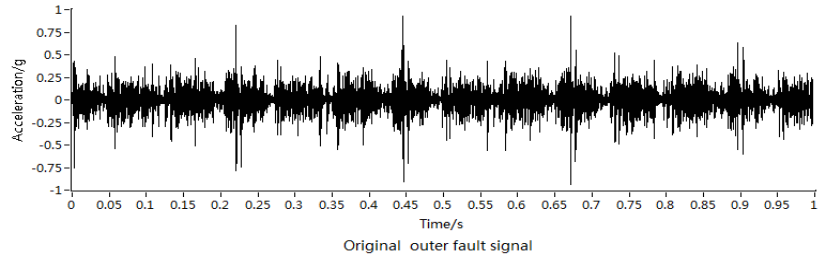

Figure 2. Original outer fault signal

Then the IMF series obtained by decomposing the preprocessed signal, and the first four IMFs chosen (Figure 4.) according to their correlation coefficients. From the Hilbert spectrum (Figure 5.) of the IMFs chosen, the real fault frequency obtained ranges from $47 \mathrm{~Hz}$ to $50 \mathrm{~Hz}$, which is very closely to its theoretical fault frequency $47.5558 \mathrm{~Hz}$ calculated by equation (8). So the fault position must be the outer ring.

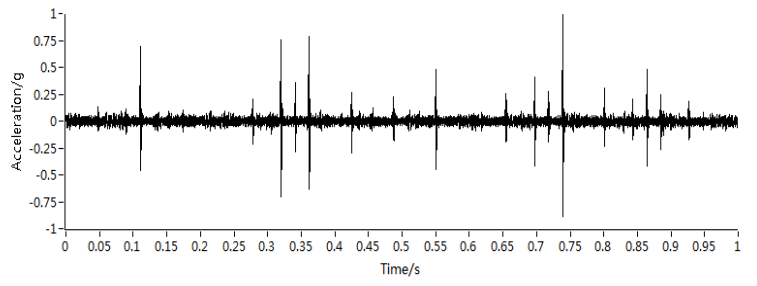

Preprocessed outer fault signal

Figure 3. Preprocessed outer fault signal
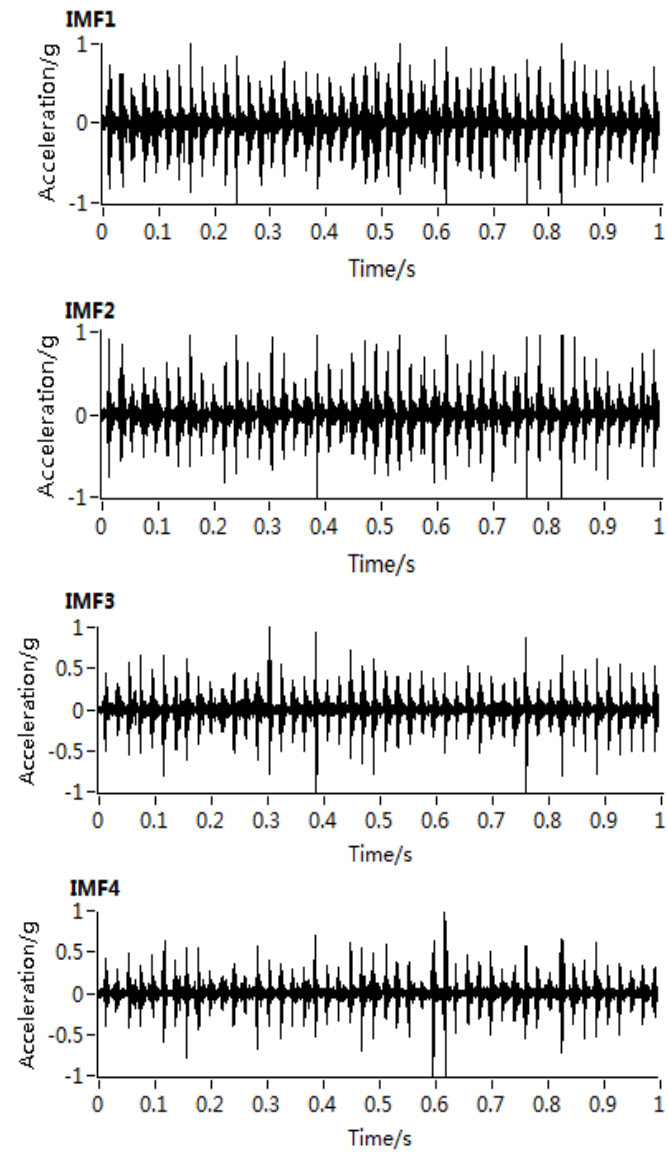

Figure 4. First four IMFs of outer ring

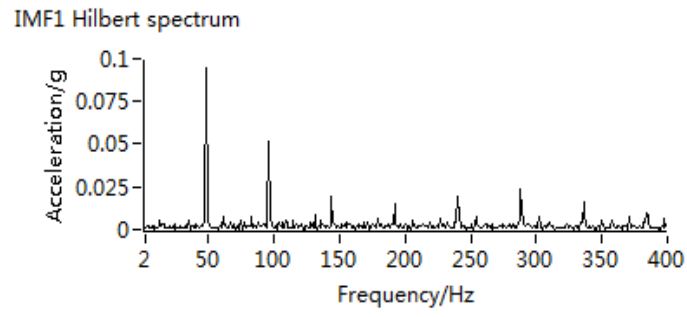

IMF2 Hilbert spectrum

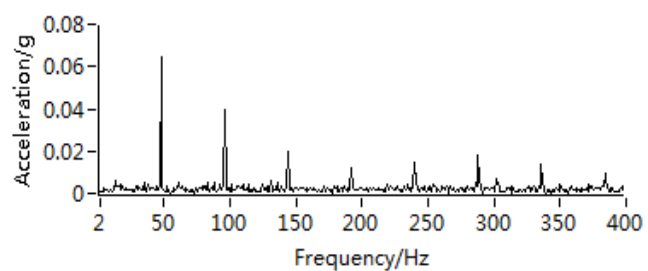

IMF3 Hilbert spectrum

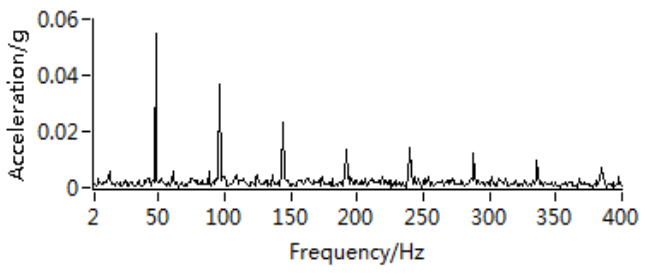

IMF4 Hilbert spectrum

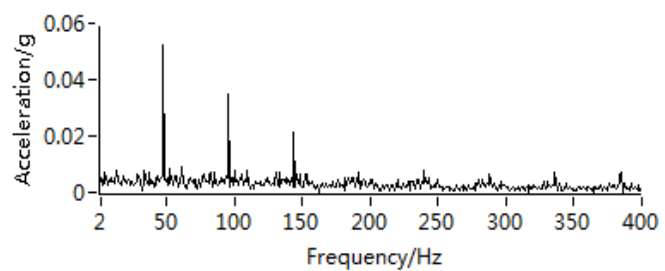

Figure 5. Hilbert spectrum of IMFs chosen

B.

\section{Inner ring fault diagnosis}

The fault signal collected (Figure 6.) of inner ring, which has been preprocessed by MED mentioned above shown in Figure 7.With the methods above, the first four IMFs of inner ring chosen (Figure 8.) according to their correlation coefficients. From the Hilbert spectrum (Figure 9.) of the IMFs chosen, the real fault frequency obtained range from $71 \mathrm{~Hz}$ to $73 \mathrm{~Hz}$, which is very closely to its

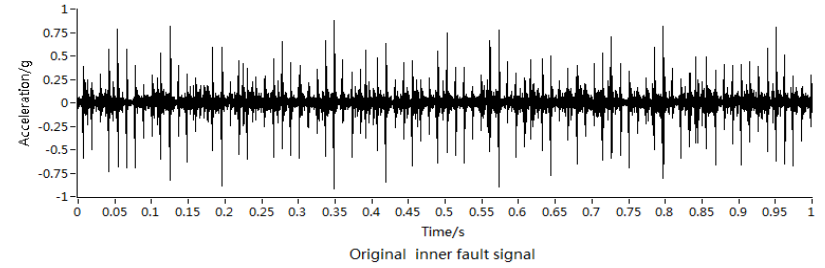

Figure 6. Original inner fault signal

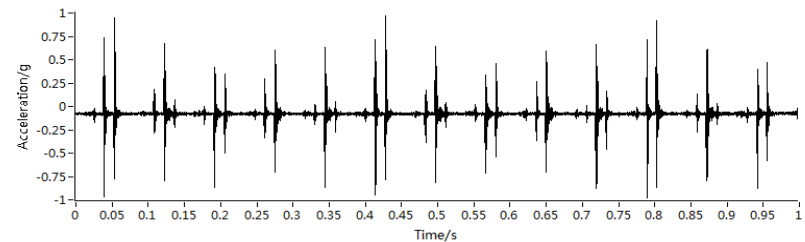

Preprocessed inner fault signal

Figure 7. Preprocessed outer fault signal 
theoretical fault frequency $72.4442 \mathrm{~Hz}$ calculated by equation (9). So the fault position must be the inner ring.
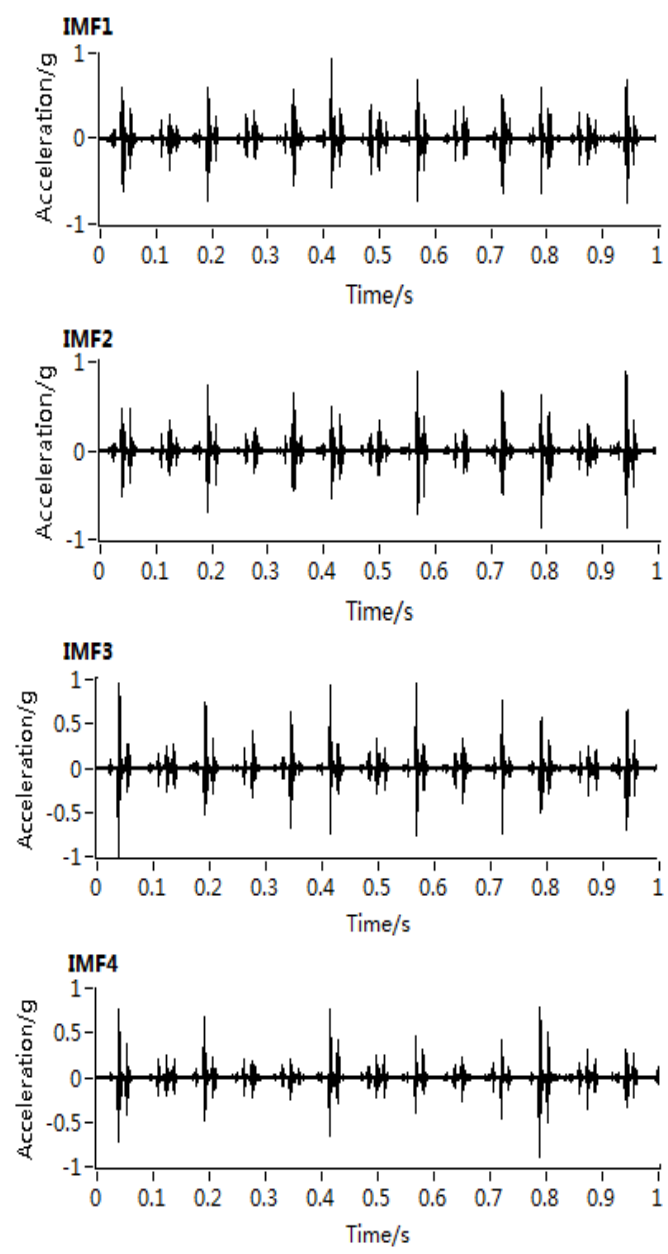

Figure 8. First four IMFs of inner ring

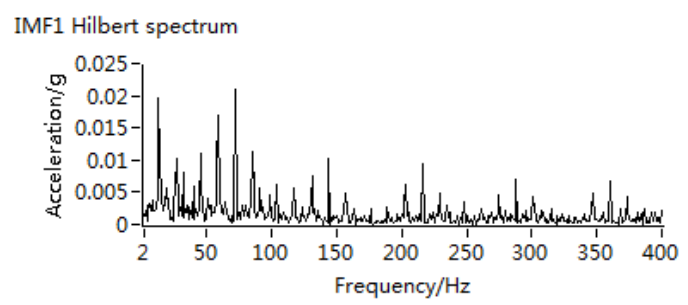

IMF2 Hilbert spectrum

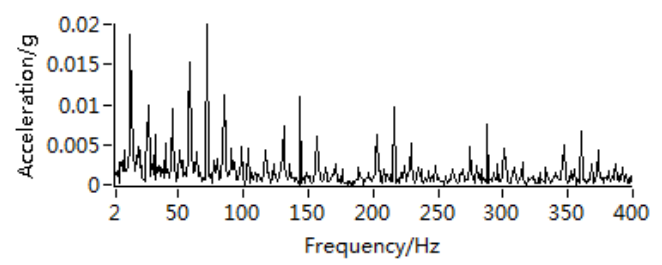

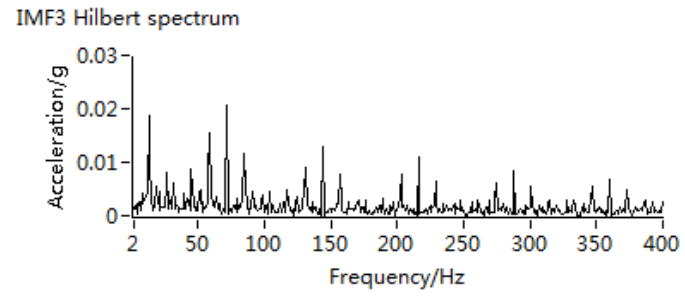

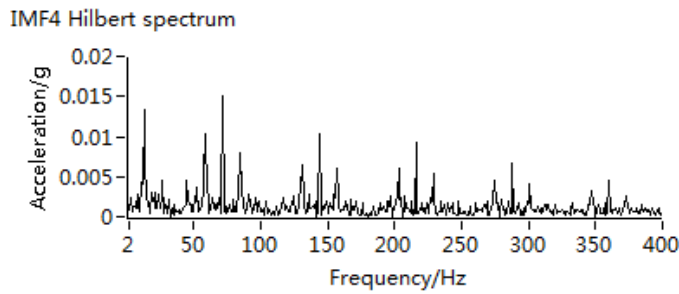

Figure 9. Hilbert spectrum of IMFs chosen

\section{CONCLUSIONS}

As known, it's very difficult to extract the effective components of early rolling bearing fault signal under the strong noise background. In this study, minimum entropy deconvolution (MED) combined with HHT applied to identify the fault position accurately of early rolling bearing. Lots of experimental results show that MED can effectively restore the weak shock signal under the strong noise condition. What's more, the endpoint effect the MED can be effectively restrained, which makes us find the fault frequency quickly and accurately. Comparing the real fault frequency obtained by the Hilbert spectrum of IMFs chosen with the theoretical failure frequency, the fault position can be identified accurately, which will greatly promoted the development of early fault diagnosis of rolling bearing technology.

\section{REFERENCES}

[1] Hui Song, Haojie Chen. The rolling bearing fault diagnosis research Based on the Morlet wavelet transform of spectral kurtosis and the empirical mode decomposition method [J]. Journal of China printing and packaging, 2012, 01(04):35-39

[2] Hongchao Wang, Jin Chen, Guangming Dong, Feiyun Cong . The application of the rolling bearing fault feature extraction of resonance demodulation method based on fast kurtogram $[\mathrm{J}]$. Journal of Vibration and Shock, 2013,32(01):35-48

[3] Guo Yu, ZHNEG Hua-wen, GAO Yan, WU Tao. The envelope analysis of rolling bearing Based on kurtogram [J]. Journal of Vibration Test and Diagnosis, 2011,31(04):517-521

[4] Lingsong He, Weihua LI. The envelope detection analysis Based on Morlet wavelet [J]. Journal of vibration engineering, 2011,31(04):517-521

[5] MENG Zong, LI Shan-shan. Rolling bearing fault diagnosis Based on wavelet of improved threshold denoising and HHT [J]. Journal of Vibration and Shock, 2013:32(14):204-214

[6] Hui Li, Licheng Zhang, Haiqi Zhang, Liwei Tang. The application of the energy spectrum of the Hilbert Huang transform in bearing fault diagnosis $[\mathrm{J}]$. Journal of Ordnance Engineering College, 2005, 17(04):37-40

[7] Geng Xiaodong. The application of HHT method in bearing fault diagnosis [J]. Journal of Shanxi University of Technology, 2012, 28(04):9-13

[8] Z.K. Peng, Peter W. Tse, F.L. Chub. A comparison study of improved Hilbert-Huang transform and wavelet transform: Application to fault diagnosis for rolling bearing [J]. Mechanical Systems and Signal Processing, 2005, 974-988 
[9] Xufeng Wang. The pipe guided wave signal processing Based on minimum Entropy Deconvolution [J].Journal of HeilongjiangScience and Technology Information, 2013,22:93-95

[10] Zhichuan Liu, Liwe Tang, Lijun Cao. Fault feature extraction of rolling bearing Based on MED and FSK [J]. Journal of
[11] Hongchao Wang, Jin Chen, Guangming Dong. Rolling bearing fault feature extraction Based on mi minimum Entropy Deconvolution and sparse decomposition [J]. Journal of mechanical engineering, 2013,49(1):88-94

[12] Wiggins $\mathrm{R}$ A. Minimum entropy deconvolution [J]. Geoexploration, 1978, 16(1):21-25. 